\title{
Parathyroid Perils: Efficiently Investigating Hypercalcaemia for Malignancy
}

\section{$\operatorname{Dr}$ T.Robbins (SpR) Dr R.Mahto (Cons)}

\section{Case Background}

69 year old man presented to hospital with acute hypercalcaemia and renal failure.

Past history of nephrectomy for a benign renal tumour and thyroidectomy for thyroid carcinoma

Presentation adjusted calcium was $2.70 \mathrm{mmol} / \mathrm{L}$ with an incompletely suppressed parathyroid hormone level of $0.6 \mathrm{pmol} / \mathrm{L}$.

Hypercalcaemia was resistant to both intravenous fluids and bisphosphonates, rising to $3.20 \mathrm{mmol} / \mathrm{L}$.

Given his previous cancer, hypercalcaemia of malignancy was strongly suspected.

CT scan demonstrated splenomegaly and small volume lymphadenopathy.

Axillary lymph node biopsy done from metabolically active lymph node on PET scan demonstrated CD20 negative angioimmunoblastic lymphoma, which was treated successfully with chemotherapy.

PTH-rp level returned as $<1.0 \mathrm{pmol} / \mathrm{L}$ over 6 weeks later.

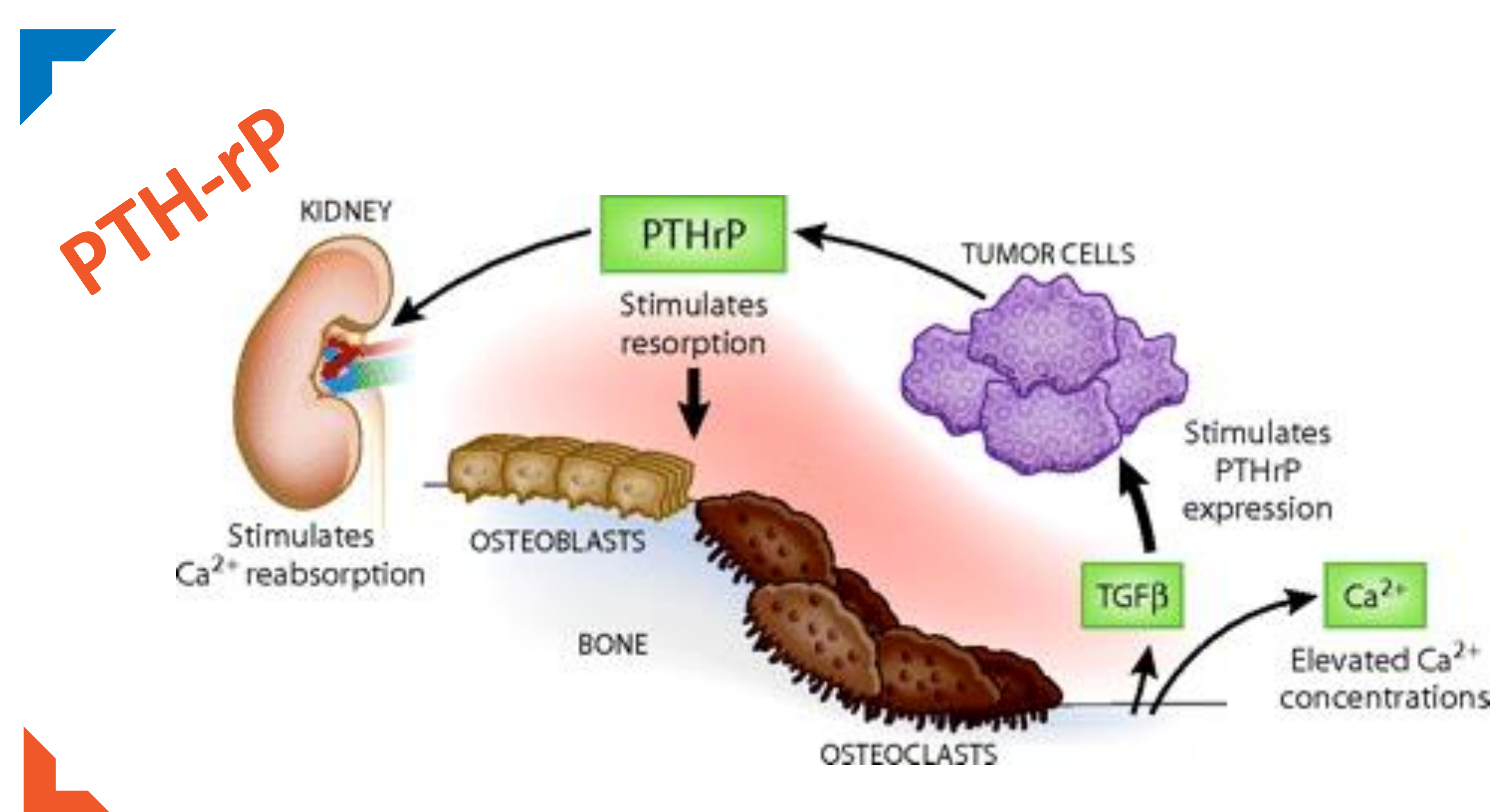

Incompletely suppressed parathyroid level in the context of hypercalcaemia is difficult to interpret. The leading cause of hypercalcaemia in the inpatient setting is malignancy. Malignancies are typically associated with an elevated PTH-rp. Importantly lymphoma can present with hypercalcaemia and either a normal or elevated PTH-rp. This case demonstrates that whilst PTH-rp level has a role in investigating hypercalcaemia with incompletely suppressed PTH, its use may be limited.

Thinking Practically...

- The complex preparation of PTH-rp samples is critical for accurate analysis, requiring strict temperature control and decanted containers.

- PTH-rp sampling often incorporates significant delays through rejected samples and complex laboratory processing.

- Lymphoma can drive hypercalcaemia through PTH-rp independent mechanisms, both through direct bone effects or 1,25-dihydroxyvitamin D production.

We therefore argue increased emphasis should be placed on the measurement of 1,25-dihydroxyvitamin $D$ to help reduce delays in diagnosing the aetiology of hypercalcaemia when clinical and radiological picture do not elicit the diagnosis readily.

\section{Potential for delays in the investigation and management of hypercalcaemia}

Potential of lymphoma to present with a normal PTH-rp

- Importance, and under-recognised use of measuring 1,25-dihydroxyvitamin D levels

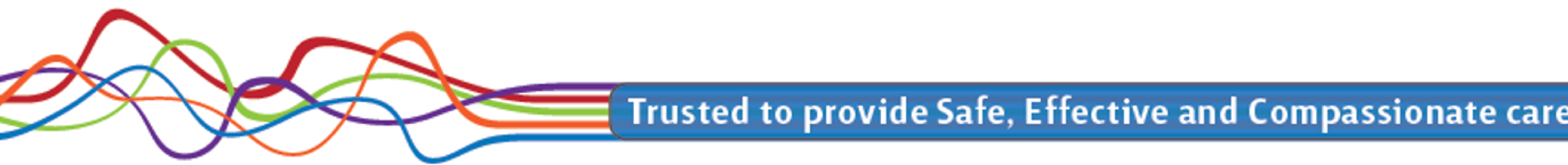

